\title{
A toolkit for MSDs prevention - WHO and IEA context
}

\author{
David C Caple** \\ Past President of the International Ergonomics Association, P.O. Box 2135, Ivanhoe East, Victoria, Australia
}

\begin{abstract}
Many simple MSD risk management tools have been developed by ergonomists for use by workers and employers with little or no training to undertake injury prevention programs in their workplace. However, currently there is no "toolkit" which places such tools within an holistic, participative ergonomics framework and provides guidance on how best to use individual tools. It is proposed that such an holistic approach should entail initial analysis and evaluation of underlying systems of work and related health and performance indicators, prior to focusing in assessment of MSD risks stemming from particular hazards. Depending on the context, more narrowly focused tools might then be selected to assess risk associated with jobs or tasks identified as problematic. This approach ensures that biomechanical risk factors are considered within a broad context of organizational and psychosocial risk factors. This is consistent with current research evidence on work- related causes of MSDs
\end{abstract}

Keywords: Holistic approach, MSD tools, systems approach, biomechanical risks, participatory ergonomics

\section{Introduction}

The International Ergonomics Association (IEA) and the World Health Organization (WHO) have identified a need for simple tools for use by small and medium sized enterprises, particularly in developing countries. These tools should enable workers and employers to implement injury prevention controls within their workplace to address MSD risks.

The users of these tools may have literacy and language difficulties requiring the style and presentation of the ergonomics information to be simple and illustrative.

A broad systems approach to ergonomics interventions has been provided in the IEA / ICOH joint publication called "Ergonomics Guidelines for Occupational Health Practice in Industrially Developing Countries, 2010". The participative approach to address actual workplace risks is outlined in the ILO/ IEA publication "Ergonomics Checkpoints". This tool utilizes positive workplace solutions to share and develop interventions using participative processes.
The use of tools to address MSD risks should be promoted within a broad holistic context. Ergonomics is a multi-factorial science that includes physical, cognitive and work organizational risk factors. Models are required to place the MSD tools within a context that can be used within the workplace.

\section{Context for Using MSD tools}

The application of MSD tools will need to take into account a range of contexts. These contexts may include different industry sectors or work environments. Whilst generic MSD tools may address the risk factors common regardless of application, more targeted tools will be required for specific application areas. For example the tools for agriculture in developing countries could address different risk factors to tools for office based work.

Managers and business owners see their workplace within a holistic framework. They look at MSD risks to workers within a broad context of risk to their business. For example, if the worker is injured

\footnotetext{
* E-mail: editorial@iospress.nl. Check if the checkbox in menu Tools/Options/Compatibility/Lay out footnotes like Word 6.x/95/97 is selected if you make a footnote for the corresponding author.
} 
and unable to work there are direct impacts on the business. The measure of success for their business is focused on the outputs they provide. For example, good health outcomes for patients, satisfied customers for products or services, quality educational outcomes for schools children. Hence their measure of success for their MSD prevention program is within this holistic context.

Decisions that are made for expenditure for workplace changes are also assessed within this holistic context. The "return on investment" is one term used to describe how managers assess how far they go in their MSD prevention program. Whilst ergonomics analysis may highlight advantages to the workers of particular risk controls, the decision of which controls are implemented is assessed within this broader context. The IEA definition of ergonomics focuses on enhancing human wellbeing as well as systems performance. Hence the outcomes in using the MSD tools should also address both of these issues.

The structure and style of the tools should encourage the workers and managers to explore the multiple potential risk factors that may be present, together with their interaction affects.

Before commencing the use of the tools within the workplace the managers and workers need to be involved in consultation to clarify their respective expectations. For example the workers may wish to use the tool to identify systemic changes to their work environment to address MSD risks whilst managers may wish to focus on the skills and training of the workers to work safely within the existing environment. The tools need to be flexible to enable both expectations to be met.

If changes to the work environment are to be made they need to be addressed not just with the current work systems but also future requirements. Consultation is required between workers and managers to understand what the plan for this workplace is over a long term timeframe, for example two to five years. This will enable greater scope for holistic changes to be implemented.

\section{Multi disciplinary Models}

Ergonomics utilizes multidisciplinary models to MSD prevention including physical, cognitive and work organizational risk factors.

Consequently the MSD prevention tools should guide the user to assess these within the context of their specific workplace requirements. Sustainability of the risk control plan will be directly impacted by the holistic approach to this risk assessment.

Participatory ergonomics methods involve the workers directly in the MSD risk identification, assessment and the determination of effective risk controls. The tools need to be structured to guide a participatory approach to be adopted.

An alternative is the expert driven approach where the ergonomist undertakes the MSD risk assessment utilizing their knowledge and expertise. The WHO and IEA require tools that are not dependent on an expert being available. They should be flexible to not always be dependent on a risk assessment approach. The MSD tools need to be structured to enable workers and managers to understand the risk factors using simple language, concepts and presentation styles. If the risk controls are known then the tool should guide them to the simple risk control. If the risk control is not known or agreed then a more detailed risk assessment may be required to understand the risk factors that need to be controlled.

\section{Holistic systems approach}

This systems approach reflects the methodology to be followed by the ergonomist or the leader of the MSD prevention program within the workplace.

The key steps in this approach include;

1. Engage with managers, business owners and work representatives to determine their expectations and measures of success

2. Conduct a holistic systems analysis of the work areas to be assessed to determine the key performance requirements.

3. Utilize the MSD tool to assess the risk factors for the tasks using a participatory approach.

4. Determine which physical, cognitive and work organizational risks that needs to be controlled.

5. Consult with the key stakeholders to develop an Action Plan to eliminate or reduce these risks.

6. Conduct follow up assessments using the Tools and consultation processes to determine if the measures were successful.

This systems approach will optimize the benefits in using the tools to address the MSD risks within the workplaces. 


\section{Tool design requirements}

The structure and content of the MSD risk management tools need to enable the worker or manager to step by step follow the holistic approach to ergonomics assessment and risk control.

After the initial discussions with key stakeholders the tools should encourage the users to consider;

1. Data in relation to incidents, injuries and key performance measures used. For Example, surveys on MSD discomfort reported by the workers, production data and quality defects

2. Observation of the work tasks to focus on the postures and movements of the workers within the context of their work duties

3. Consultation with workers and others who are involved with the work area. This may include maintenance or cleaners who interface with the workplace. This may include open ended questions.

For example a question that encourages the workers to describe what it is about their job that they think results in MSD discomfort is easier for them to answer rather than a question about specific postures and movements.

Once these are completed there may be a requirement to collect more quantitative and qualitative data. This would include measurements of the workplace and the tasks being undertaken. Photos and videos of a sample of different workers undertaking the same tasks are also useful. They enable subsequent analysis by the users of the MSD tools. They are also useful to show during a focus group or similar to encourage further detailed observations of the work environment by the workers and managers.

Qualitative data can be collected by surveys and direct discussions with the workers as part of the MSD tool. The biomechanical component of the MSD tool needs to reflect the ergonomics research in relation to MSD causation and prevention. Specific MSD tools may be appropriate for the main MSD areas. This could be lower back; neck and shoulders or arms and hands. Alternatively they could guide the user to each area from an initial broader risk analysis step.

The cognitive risks associated with the job design and workload expectations will need inclusion within the MSD tool to enable consideration of the mental workload and the potential relationship to the MSD risks.

The work organizational risk factors need inclusion to assess the psychosocial risks arising from how the work is organized. This needs to include not just the direct impact on the worker for example on cumulative fatigue but also interactive affects for example of supervision styles and interpersonal conflict that may increase static load symptoms in the neck and shoulders.

\section{Conclusion}

The WHO and the IEA have both identified that workers and employers, particularly in developing countries need access to practical and simple tools to enable them to identify and control MSD risks within their workplace.

Whilst access to an ergonomist or technical specialist is desirable to assist them to implement an ergonomics program within their workplace, this is not always possible. A toolkit explaining how to use a set of simple MSD risk management tools was identified as a resource required for local level programs managed by the workers and managers together. This toolkit needs to address the multiple and interacting hazards and risk factors influencing MSD risk. This should include organizational and psychosocial hazards as well as the more widely recognized physical hazards.

Development of this toolkit will be followed by its implementation and evaluation in both developing and developed countries. The IEA and WHO are keen for the toolkit to be easily accessible to encourage their use at a global level.

\section{References}

[1] International Ergonomics Association, 2010, Ergonomics Guidelines for Occupational Health Practice in Industrially Developing Countries. Joint ICOH and IEA Publication.

[2] International Ergonomics Association www.iea.cc .Definition of Ergonomics

[3] International Labor Organization, 2009, Ergonomics Checkpoints; $2^{\text {nd }}$ Edition. ILO, Geneva 